$\stackrel{W}{=}$

Global burnals Inc.

औf

GLObal JOURnal of MedicAl ReSEARCH: K

INTERDISCIPLINARY

Volume 20 Issue 12 Version 1.0 Year 2020

Type: Double Blind Peer Reviewed International Research Journal

Publisher: Global Journals

Online ISSN: 2249-4618 \& Print ISSN: 0975-5888

\title{
Effect of Alcohol Disinfection on the Handle and Blade of Vegetables Knives by using ATP Inspection and Microbial Stamp Test
}

By Naomi Katayama, Akemi Ito, Mayumi Hirabayashi, Natuki Sasaki \& Moe Inuzuka

Nagoya Women's University

Abstract- To prevent food poisoning, we focused on kitchen vegetable knives, which are likely to cause secondary contamination, and conducted hygiene inspections to obtain results. The values after cooking and after washing, and after washing and after 70\%spraying alcohol compared using the ATP wiping test and the microbial stamp test (selective medium: general bacteria, Escherichia coli, Staphylococcus aureus, Salmonella, Vibrio parahaemolyticus). As a result, the ATP test value decreased statistically significantly and became 100 or less after $70 \%$ alcohol sprayings. However, not all of the bacterial counts in the selective medium were statistically significantly different even after $70 \%$ alcohol sprayings. The reason the data was not clear due to $70 \%$ alcohol spray conditions. It may be advisable to perform a microbiological stamp test after applying $70 \%$ alcohol spray multiple times instead of once to a fully moistened knife handle and blade. In the future, we would like to consider the handling of more hygienic cooking utensils by further changing the $70 \%$ alcohol spraying conditions and conducting, Microbiological tests using a selective medium.

Keywords: ATP test, microbial stamp test, the handle of the knife, the blade of the knife, alcohol disinfection.

GJMR-K Classification: NLMC Code: QW 51

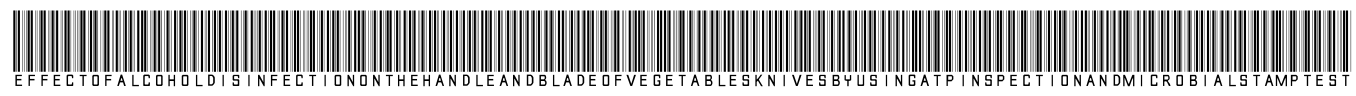

Strictly as per the compliance and regulations of:

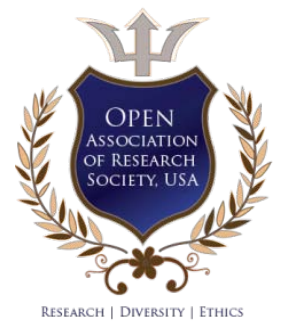

(c) 2020. Naomi Katayama, Akemi Ito, Mayumi Hirabayashi, Natuki Sasaki \& Moe Inuzuka. This is a research/review paper, distributed under the terms of the Creative Commons Attribution-Noncommercial 3.0 Unported License http://creativecommons.org/licenses/by-nc/3.0/), permitting all non-commercial use, distribution, and reproduction in any medium, provided the original work is properly cited. 


\title{
Effect of Alcohol Disinfection on the Handle and Blade of Vegetables Knives by using ATP Inspection and Microbial Stamp Test
}

\author{
Naomi Katayama ${ }^{\alpha}$, Akemi Ito ${ }^{\sigma}$, Mayumi Hirabayashi ${ }^{\rho}$, Natuki Sasaki ${ }^{\omega}$ \& Moe Inuzuka ${ }^{*}$
}

\begin{abstract}
To prevent food poisoning, we focused on kitchen vegetable knives, which are likely to cause secondary contamination, and conducted hygiene inspections to obtain results. The values after cooking and after washing, and after washing and after $70 \%$ spraying alcohol compared using the ATP wiping test and the microbial stamp test (selective medium: general bacteria, Escherichia coli, Staphylococcus aureus, Salmonella, Vibrio parahaemolyticus). As a result, the ATP test value decreased statistically significantly and became 100 or less after $70 \%$ alcohol sprayings. However, not all of the bacterial counts in the selective medium were statistically significantly different even after $70 \%$ alcohol sprayings. The reason the data was not clear due to $70 \%$ alcohol spray conditions. It may be advisable to perform a microbiological stamp test after applying $70 \%$ alcohol spray multiple times instead of once to a fully moistened knife handle and blade. In the future, we would like to consider the handling of more hygienic cooking utensils by further changing the $70 \%$ alcohol spraying conditions and conducting, Microbiological tests using a selective medium.
\end{abstract}

Keywords: ATP test, microbial stamp test, the handle of the knife, the blade of the knife, alcohol disinfection.

\section{INTRODUCTION}

$\longrightarrow$ leaning and disinfecting cooking utensils, and cleaning and disinfecting hands, avoid the risk of food poisoning. Cleaning and disinfecting kitchen knives, which often come into contact with food, helps prevent secondary contamination. Many researchers have achieved hygiene management in hospitals and other kitchens through hygiene education ${ }^{1,2,3,4)}$. In particular, hygiene management using the ATP wiping test made it possible to create an easy-to-understand and hygienic environment by expressing invisible microorganisms as ATP values ${ }^{5,6,7,8)}$. In the past, we also reported the results of hygiene tests on kitchen utensils using ATP wiping test $t^{9,10,11)}$. Since it is impossible to know what kind of bacteria are present in the ATP wiping test, a more detailed hygiene test can obtain by examining food poisoning bacteria using a microbial selection medium. Therefore, in this study, we report the

Author a $\omega ¥:$ Nagoya Women's University, Nagoya City, Japan.

Author $\alpha \sigma \rho$ : Graduate School of Nagoya Women's University, Nagoya City, Japan.

Author a: Department of Otorhinolaryngology, Nagoya University Graduate School of Medicine, Nagoya, Japan.

Corresponding Author a: Nagoya Women's University, Nagoya City, Japan.e-mail: naomik@nagoya-wu.ac.jp hygiene of the handle and blade of a vegetable knife by performing an ATP wiping test and a microbial stamp simultaneously after cooking, cleaning, and spraying alcohol.

\section{Materials and Methods}

a) Hygiene tests on Kitchen knife

Hygiene tests on six vegetable knives performed using the ATP test kit (KIKKOMAN CO., Ltd.) and the microbial stamp test kit (NISSUI Co., Ltd.).

b) ATP wiping tests

ATP wiping tests performed on the handles and blades of 6 meat and fish knives. The ATP test was performed by the inspector three times immediately after cooking, after washing, and after $70 \%$ spraying alcohol. The inspector recorded the ATP test results.

c) Microbial stamp test

And the inspector performed a microbial stamp test as same as ATP tests (three times: after cooking, after washing, and after spraying alcohol). The microbial stamp was then cultured in an incubator at 38 degrees for three days. After culturing, microbial stamps were counted and recorded by the inspector.

\section{d) Statistical processing}

The results obtained compared using statistical methods. Compared data were subjected to an F test to determine whether to use a parametric test or nonparametric test. When there is no difference in the $\mathrm{F}$ test, the presence or absence of a significant difference was confirmed using the student t-test with or without a correspondence. If there was a difference in the $\mathrm{F}$ test, the presence or absence of a significant difference was confirmed using the Wilcoxon test with a pair or the Mann-Whitney test without correlation.

\section{Results}

a) Vegetable knife: ATP results and microorganisms stamp test results of Alcohol disinfection

i. ATP test results of vegetable kitchen knife handle and blade

The ATP test values were lower on both the handle and blade of vegetable knives after washing than after cooking, and after spraying $70 \%$ alcohol than after 
washing. After spraying alcohol, the ATP value of both the handle and blade of the knife was 100 or less. It judged that the handle and blade of the vegetable knife were in a hygienic condition (See Table 1 and Table 2).

Table1. ATP test value and statistical processing result of Kitchen knife Handle

\begin{tabular}{|c|c|c|c|c|}
\hline \multirow[b]{2}{*}{ For vegetables } & \multicolumn{2}{|c|}{ No alcohol treatment } & \multicolumn{2}{|c|}{ Alcohol treatment } \\
\hline & Before washing & After washing & After washing & After alcohol \\
\hline 1 & 159550 & 4828 & 4828 & 59 \\
\hline 2 & 2294 & 558 & 558 & 23 \\
\hline 3 & 37952 & 6919 & 6919 & 8 \\
\hline 4 & 12836 & 3691 & 3691 & 77 \\
\hline 5 & 13009 & 4260 & 4260 & 28 \\
\hline 6 & 2531 & 2813 & 2813 & 18 \\
\hline Average value & 38028.7 & 3844.8 & 3844.8 & 35.5 \\
\hline SD & 60934.6 & 2120.4 & 2120.4 & 26.6 \\
\hline $\begin{array}{l}\mathrm{F} \text { test } \\
\text { Student-t* }\end{array}$ & \multicolumn{2}{|c|}{$\mathrm{P}=0.0001^{* *}$} & \multicolumn{2}{|c|}{$\mathrm{P}=0.0001^{* *}$} \\
\hline Wilcoxon & \multicolumn{2}{|c|}{$\mathrm{P}=0.046 *$} & \multicolumn{2}{|c|}{$\mathrm{P}=0.028^{*}$} \\
\hline $\begin{array}{l}\text { F test } \\
\text { Student-t* }\end{array}$ & \multicolumn{4}{|c|}{$\mathrm{P}=0.0001^{* *}$} \\
\hline Wilcoxon & \multicolumn{4}{|c|}{$\mathrm{P}=0.028^{*}$} \\
\hline
\end{tabular}

*Paired Student-t test $* \mathrm{P}<0.05,{ }^{* *} \mathrm{P}<0.01$

Table2. ATP test value and statistical processing result of Kitchen knife Blade

\begin{tabular}{|c|c|c|c|c|}
\hline \multirow[b]{2}{*}{ For vegetables } & \multicolumn{2}{|c|}{ No alcohol treatment } & \multicolumn{2}{|c|}{ Alcohol treatment } \\
\hline & Before washing & After washing & After washing & After alcohol \\
\hline 1 & 157036 & 163 & 163 & 45 \\
\hline 2 & 183 & 1232 & 1232 & 21 \\
\hline 3 & 4635 & 91 & 91 & 47 \\
\hline 4 & 7962 & 58 & 58 & 7 \\
\hline 5 & 382923 & 664 & 664 & 8 \\
\hline 6 & 1102 & 529 & 529 & 15 \\
\hline Average value & 92306.8 & 456.2 & 456.2 & 23.8 \\
\hline SD & 155082.7 & 453.5 & 453.5 & 17.9 \\
\hline $\begin{array}{l}\text { F test } \\
\text { Student-t* }\end{array}$ & \multicolumn{2}{|c|}{$\mathrm{P}-0.0001^{* *}$} & \multicolumn{2}{|c|}{$\mathrm{P}=0.0001^{* *}$} \\
\hline Wilcoxon & \multicolumn{2}{|c|}{$\mathrm{P}=0.075$} & \multicolumn{2}{|c|}{$\mathrm{P}=0.028^{*}$} \\
\hline $\begin{array}{l}\mathrm{F} \text { test } \\
\text { Student-t* }\end{array}$ & \multicolumn{4}{|c|}{$\mathrm{P}=0.0001^{* *}$} \\
\hline Wilcoxon & \multicolumn{4}{|c|}{$\mathrm{P}=0.028^{*}$} \\
\hline
\end{tabular}

b) Microbial stamp test results of vegetable kitchen knife handle and blade

\section{i. General bacteria}

A microbial stamp test (general bacteria) performed on the handle and blade of a vegetable knife. The results are shown in Tables 3 and 4 . Bacterial counts decreased after washing than after cooking and after $70 \%$ alcohol sprayings than after washing, not all were statistically significant. The number of microorganisms after spraying with $70 \%$ alcohol was not sufficiently reduced as compared with that after washing.

Table 3 Number of general bacteria on Kitchen knife Handle and statistical processing result

\begin{tabular}{|c|c|c|c|c|}
\hline \multirow[b]{2}{*}{ For vegetables } & \multicolumn{2}{|c|}{ No alcohol treatment } & \multicolumn{2}{|c|}{ "Alcohol treatment } \\
\hline & Before washing & After washing & After washing & After alcohol \\
\hline 1 & 22 & 14 & 14 & 3 \\
\hline 2 & 35 & 18 & 18 & 15 \\
\hline 3 & 41 & 20 & 20 & 20 \\
\hline 4 & 10 & 1 & 1 & 3 \\
\hline 5 & 70 & 3 & 3 & 2 \\
\hline 6 & 20 & 3 & 3 & 0 \\
\hline Average value & 33.0 & 9.8 & 9.8 & 7.2 \\
\hline SD & 21.2 & 8.5 & 8.5 & 8.2 \\
\hline F test & \multicolumn{2}{|c|}{$\mathrm{P}=0.021^{*}$} & \multirow{3}{*}{\multicolumn{2}{|c|}{$\begin{array}{l}\mathrm{P}=0.473 \\
\mathrm{P}=0.206\end{array}$}} \\
\hline Student-t* & \multirow{2}{*}{\multicolumn{2}{|c|}{$\mathrm{P}=0.028^{*}$}} & & \\
\hline Wilcoxon & & & & \\
\hline F test & \multirow{2}{*}{\multicolumn{4}{|c|}{$\mathrm{P}=0.018^{*}$}} \\
\hline Student-t* & & & & \\
\hline Wilcoxon & \multicolumn{4}{|c|}{$\mathrm{P}=0.028^{*}$} \\
\hline
\end{tabular}


Table 4 Number of general bacteria on Kitchen knife Blade and statistical processing

\begin{tabular}{|c|c|c|c|c|}
\hline \multirow[b]{2}{*}{ For vegetables } & \multicolumn{2}{|c|}{ "No alcohol treatment } & \multicolumn{2}{|c|}{ "Alcohol treatment } \\
\hline & Before washing & After washing & After washing & After alcohol \\
\hline 1 & 49 & 1 & 1 & 4 \\
\hline 2 & 0 & 13 & 13 & 26 \\
\hline 3 & 8 & 17 & 17 & 2 \\
\hline 4 & 41 & 59 & 59 & 0 \\
\hline 5 & 198 & 48 & 48 & 21 \\
\hline 6 & 0 & 44 & 44 & 0 \\
\hline Average value & 49.3 & 30.3 & 30.3 & 8.8 \\
\hline SD & 75.8 & 23.1 & 23.1 & 11.6 \\
\hline F test & \multicolumn{2}{|c|}{$\mathrm{P}=0.021^{*}$} & \multirow{2}{*}{\multicolumn{2}{|c|}{$\begin{array}{l}\mathrm{P}=0.473 \\
\mathrm{P}=0.206\end{array}$}} \\
\hline Student- $t^{*}$ & & & \\
\hline Wilcoxon & \multirow{3}{*}{\multicolumn{4}{|c|}{$\mathrm{P}=0.0001^{* *}$}} \\
\hline F test & & & & \\
\hline Student-t* & & & & \\
\hline Wilcoxon & \multicolumn{4}{|c|}{$\mathrm{P}=0.138$} \\
\hline
\end{tabular}

\section{ii. Escherichia Coli (E Coli)}

The number of E. coli performed on the handle and blade of a vegetable knife. The results shown in Tables 5 and 6 . Bacterial counts decreased after washing than after cooking and after $70 \%$ alcohol sprayings than after washing, not all were statistically significant. The number of microorganisms on the handle of the kitchen vegetable knife did not decrease statistically significantly.

Table 5 Number of E. coli on Kitchen knife Handle and statistical processing result

\begin{tabular}{|c|c|c|c|c|}
\hline \multirow[b]{2}{*}{ For vegetables } & \multicolumn{2}{|c|}{ No alcohol treatment } & \multicolumn{2}{|c|}{ Alcohol treatment } \\
\hline & Before washing & After washing & After washing & After alcohol \\
\hline 1 & 8 & 14 & 14 & 3 \\
\hline 2 & 1 & 3 & 3 & 0 \\
\hline 3 & 34 & 0 & 0 & 11 \\
\hline 4 & 1 & 0 & 0 & 0 \\
\hline 5 & 0 & 1 & 1 & 0 \\
\hline 6 & 0 & 0 & 0 & 0 \\
\hline Average value & 7.3 & 3.0 & 3.0 & 2.3 \\
\hline SD & 13.4 & 5.5 & 5.5 & 4.4 \\
\hline F test & \multicolumn{2}{|c|}{$\mathrm{P}=0.024^{*}$} & \multirow{3}{*}{\multicolumn{2}{|c|}{$\begin{array}{l}\mathrm{P}=0.301 \\
\mathrm{P}=0.826\end{array}$}} \\
\hline Student-t* & \multirow{2}{*}{\multicolumn{2}{|c|}{$\mathrm{P}=0.787$}} & & \\
\hline Wilcoxon & & & & \\
\hline F test & \multirow{2}{*}{\multicolumn{4}{|c|}{$\mathrm{P}=0.008^{* *}$}} \\
\hline Student-t* & & & & \\
\hline Wilcoxon & \multicolumn{4}{|c|}{$\mathrm{P}=0.068$} \\
\hline
\end{tabular}

Table 6 Number of E. coli on Kitchen knife Blade and statistical processing result

\begin{tabular}{|c|c|c|c|c|}
\hline \multirow[b]{2}{*}{ For vegetables } & \multicolumn{2}{|c|}{ No alcohol treatment } & \multicolumn{2}{|c|}{ "Alcohol treatment } \\
\hline & Before washing & After washing & After washing & After alcoho \\
\hline 1 & 35 & 0 & 0 & 4 \\
\hline 2 & 2 & 21 & 21 & 2 \\
\hline 3 & 66 & 78 & 78 & 0 \\
\hline 4 & 4 & 1 & 1 & 0 \\
\hline 5 & 55 & 3 & 3 & 1 \\
\hline 6 & 1 & 1 & 1 & 0 \\
\hline Average value & 27.2 & 17.3 & 17.3 & 1.2 \\
\hline SD & 29.0 & 30.8 & 30.8 & 1.6 \\
\hline F test & & \multicolumn{2}{|c|}{$\mathrm{P}=0.0001^{* *}$} \\
\hline Student-t* & \multicolumn{2}{|c|}{$\mathrm{P}=426$} & \multirow{2}{*}{\multicolumn{2}{|c|}{$\mathrm{P}=0.173$}} \\
\hline Wilcoxon & & & & \\
\hline F test & \multirow{2}{*}{\multicolumn{4}{|c|}{$\mathrm{P}=0.0001^{* *}$}} \\
\hline Student-t* & & & & \\
\hline Wilcoxon & \multicolumn{4}{|c|}{$\mathrm{P}=0.043^{*}$} \\
\hline
\end{tabular}

\section{iii. Staphylococcus aureus}

Tables 7 and 8 show the results for Staphylococcus aureus. There was no statistically significant difference between the knife blade after cooking and after cleaning and after cleaning and after $70 \%$ spraying alcohol. However, the number of bacteria is decreasing. The number of bacteria on the handle of the kitchen vegetable knife is statistically significantly reduces after washing and after spraying with $70 \%$ alcohol. 
Table 7 Number of Staphylococcus aureus on Kitchen knife Handle and statistical processing result

\begin{tabular}{|c|c|c|c|c|}
\hline \multirow[b]{2}{*}{ For vegetables } & \multicolumn{2}{|c|}{ "No alcohol treatment } & \multicolumn{2}{|c|}{ Alcohol treatment } \\
\hline & Before washing & After washing & After washing & After alcohol \\
\hline 1 & 65 & 42 & 42 & 0 \\
\hline 2 & 70 & 12 & 12 & 0 \\
\hline 3 & 6 & 64 & 64 & 3 \\
\hline 4 & 1 & 3 & 3 & 0 \\
\hline 5 & 9 & 1 & 1 & 0 \\
\hline 6 & 70 & 2 & 2 & 0 \\
\hline Average value & 36.8 & 20.7 & 20.7 & 0.5 \\
\hline SD & 34.6 & 26.3 & 26.3 & 1.2 \\
\hline F test & \multicolumn{2}{|c|}{$\mathrm{P}=0.259$} & \multicolumn{2}{|c|}{$\mathrm{P}=0.0001^{* *}$} \\
\hline Student-t* & \multicolumn{2}{|c|}{$\mathrm{P}=0.425$} & \multirow{2}{*}{\multicolumn{2}{|c|}{$\mathrm{P}=0.028$}} \\
\hline Wilcoxon & & & & \\
\hline F test & \multirow{2}{*}{\multicolumn{4}{|c|}{$\mathrm{P}=0.0001^{* *}$}} \\
\hline Student-t* & & & & \\
\hline Wilcoxon & \multicolumn{4}{|c|}{$\mathrm{P}=0 . .028 *$} \\
\hline
\end{tabular}

Table 8 Number of Staphylococcus aureus on Kitchen knife Blade and statistical processing result

\begin{tabular}{|c|c|c|c|c|}
\hline \multirow[b]{2}{*}{ For vegetables } & \multicolumn{2}{|c|}{ No alcohol treatment } & \multicolumn{2}{|c|}{ Alcohol treatment } \\
\hline & Before washing & After washing & After washing & After alcohol \\
\hline 1 & 40 & 39 & 39 & 0 \\
\hline 2 & 17 & 3 & 3 & 11 \\
\hline 3 & 3 & 15 & 15 & 0 \\
\hline 4 & 1 & 3 & 3 & 0 \\
\hline 5 & 45 & 0 & 0 & 6 \\
\hline 6 & 1 & 6 & 6 & 52 \\
\hline Average value & 17.8 & 11.0 & 11.0 & 11.5 \\
\hline $\mathrm{SD}$ & 20.1 & 14.7 & 14.7 & 20.3 \\
\hline F test & \multicolumn{2}{|c|}{$\mathrm{P}=0.231$} & \multicolumn{2}{|c|}{$\mathrm{P}=0.223$} \\
\hline Student-t* & \multirow{2}{*}{\multicolumn{2}{|c|}{$\mathrm{P}=0.453$}} & \multirow{2}{*}{\multicolumn{2}{|c|}{$\mathrm{P}=0.957$}} \\
\hline Wilcoxon & & & & \\
\hline F test & \multirow{3}{*}{\multicolumn{4}{|c|}{$\begin{array}{l}\mathrm{P}=0.488 \\
\mathrm{P}=0.660\end{array}$}} \\
\hline Student-t* & & & & \\
\hline Wilcoxon & & & & \\
\hline
\end{tabular}

\section{iv. Salmonella}

The results of Salmonella shown in Tables 9 and 10. The number of bacteria decreased after washing than after cooking and after spraying $70 \%$ alcohol than after washing. However, the number of Salmonella was not statistically significantly reduced in the handle of the kitchen vegetable knife. With the knife blade, the number of Salmonella bacteria after $70 \%$ alcohol spraying was statistically significantly lower than that after cooking.

Table 9 Number of Salmonella on Kitchen knife Handle and statistical processing result

\begin{tabular}{|c|c|c|c|c|}
\hline \multirow[b]{2}{*}{ For vegetables } & \multicolumn{2}{|c|}{ No alcohol treatment } & \multicolumn{2}{|c|}{ Alcohol treatment } \\
\hline & Before washing & After washing & After washing & After alcohol \\
\hline 1 & 9 & 0 & 0 & 4 \\
\hline 2 & 1 & 0 & 0 & 0 \\
\hline 3 & 1 & 0 & 0 & 0 \\
\hline 4 & 0 & 0 & 0 & 0 \\
\hline 5 & 0 & 3 & 3 & 0 \\
\hline 6 & 0 & 0 & 0 & 0 \\
\hline Average value & 1.8 & 0.5 & 0.5 & 0.7 \\
\hline SD & 3.5 & 1.2 & 1.2 & 1.6 \\
\hline F test & \multicolumn{2}{|c|}{$\mathrm{P}=0.010^{*}$} & \multicolumn{2}{|c|}{$\mathrm{P}=0.251$} \\
\hline Student- $\mathrm{t}^{*}$ & & & \\
\hline Wilcoxon & \multicolumn{2}{|c|}{$\mathrm{P}=0.465$} & & \\
\hline F test & \multirow{2}{*}{\multicolumn{4}{|c|}{$\mathrm{P}=0.041^{*}$}} \\
\hline Student-t* & & & & \\
\hline Wilcoxon & \multicolumn{4}{|c|}{$\mathrm{P}=0.109$} \\
\hline
\end{tabular}


Table 10 Number of Salmonella on Kitchen knife Blade. and statistical processing

result

\begin{tabular}{|c|c|c|c|c|}
\hline \multirow[b]{2}{*}{ For vegetables } & \multicolumn{2}{|c|}{ No alcohol treatment } & \multicolumn{2}{|c|}{ Alcohol treatment } \\
\hline & Before washing & After washing & After washing & After alcohol \\
\hline 1 & 35 & 94 & 94 & 0 \\
\hline 2 & 1 & 0 & 0 & 0 \\
\hline 3 & 1 & 0 & 0 & 0 \\
\hline 4 & 5 & 0 & 0 & 0 \\
\hline 5 & 130 & 1 & 1 & 1 \\
\hline 6 & 1 & 0 & 0 & 0 \\
\hline Average value & 28.8 & 15.8 & 15.8 & 0.2 \\
\hline SD & 51.3 & 38.3 & 38.3 & 0.4 \\
\hline F test & \multicolumn{2}{|c|}{$\mathrm{P}=0.247$} & \multicolumn{2}{|c|}{$\mathrm{P}=0.0001^{* *}$} \\
\hline Student-t* & \multicolumn{2}{|c|}{$\mathrm{P}=0.629$} & \multirow{2}{*}{\multicolumn{2}{|c|}{$\mathrm{P}=3.17$}} \\
\hline Wilcoxon & & & & \\
\hline F test & \multirow{2}{*}{\multicolumn{4}{|c|}{$\mathrm{P}=0.0001^{* *}$}} \\
\hline Student-t* & & & & \\
\hline Wilcoxon & \multicolumn{4}{|c|}{$\mathrm{P}=0.028^{*}$} \\
\hline
\end{tabular}

\section{v. Vibrio parahaemolyticus}

The results of Vibrio parahaemolyticus shown in Tables 11 and 12. The number of bacteria decreased after washing than after cooking and after spraying $70 \%$ alcohol than after washing, but there was no statistically significant difference.

Table 11 Number of Vibrio parahaemolyticus on Kitchen knife Handleand statistical processing result

\begin{tabular}{|c|c|c|c|c|}
\hline \multirow[b]{2}{*}{ For vegetables } & \multicolumn{2}{|c|}{ No alcohol treatment } & \multicolumn{2}{|c|}{ Alcohol treatment } \\
\hline & Before washing & After washing & After washing & After alcohol \\
\hline 1 & 1 & 0 & 0 & 0 \\
\hline 2 & 71 & 0 & 0 & 1 \\
\hline 3 & 28 & 22 & 22 & 3 \\
\hline 4 & 1 & 0 & 0 & 2 \\
\hline 5 & 0 & 3 & 3 & 7 \\
\hline 6 & 0 & 0 & 0 & 0 \\
\hline Average value & 16.8 & 4.2 & 4.2 & 2.2 \\
\hline SD & 28.7 & 8.8 & 8.8 & 2.6 \\
\hline $\begin{array}{l}\text { F test } \\
\text { Student-t* }\end{array}$ & \multicolumn{2}{|c|}{$\mathrm{P}=0.006^{* *}$} & \multicolumn{2}{|c|}{$\mathrm{P}=0.005^{* *}$} \\
\hline Wilcoxon & \multicolumn{2}{|c|}{$\mathrm{P}=0.225$} & \multicolumn{2}{|c|}{$\mathrm{P}=0.715$} \\
\hline F test & \multicolumn{4}{|c|}{$\mathrm{P}=0.0001^{* *}$} \\
\hline Wilcoxon & \multicolumn{4}{|c|}{$\mathrm{P}=0.418$} \\
\hline
\end{tabular}

Table 12 Number of Vibrio parahaemolyticus on Kitchen knife Blade and statistical processing result

\begin{tabular}{|c|c|c|c|c|}
\hline \multirow[b]{2}{*}{ For vegetables } & \multicolumn{2}{|c|}{ "No alcohol treatment } & \multicolumn{2}{|c|}{ Alcohol treatment } \\
\hline & Before washing & After washing & After washing & After alcohol \\
\hline 1 & 0 & 0 & 0 & 1 \\
\hline 2 & 0 & 3 & 3 & 0 \\
\hline 3 & 0 & 0 & 0 & 0 \\
\hline 4 & 1 & 0 & 0 & 0 \\
\hline 5 & 40 & 3 & 3 & 0 \\
\hline 6 & 0 & 2 & 2 & 0 \\
\hline Average value & 6.8 & 1.3 & 1.3 & 0.2 \\
\hline SD & 16.3 & 1.5 & 1.5 & 0.4 \\
\hline $\begin{array}{l}\text { F test } \\
\text { Student-t* }\end{array}$ & \multicolumn{2}{|c|}{$\mathrm{P}=0.001^{* *}$} & \multicolumn{2}{|c|}{$\mathrm{P}=0.003^{* *}$} \\
\hline Wilcoxon & \multicolumn{2}{|c|}{$\mathrm{P}=1.000$} & \multicolumn{2}{|c|}{$\mathrm{P}=0.144$} \\
\hline $\begin{array}{l}\text { F test } \\
\text { Student-t* }\end{array}$ & \multicolumn{4}{|c|}{$\mathrm{P}=0.0001^{* *}$} \\
\hline Wilcoxon & \multicolumn{4}{|c|}{$\mathrm{P}=0.423$} \\
\hline
\end{tabular}

\section{Discussion}

This time, the ATP value became 100 or less after spraying $70 \%$ alcohol, and the handle and blade of the knife became hygienic. However, the results of the microbial stamp test using the selective medium showed that the number of bacteria did not decrease sufficiently even after spraying with $70 \%$ alcohol. The bactericidal effect of alcohol spray differed depending on the type of bacteria. After cleaning, wipe off the water sufficiently and spray $70 \%$ alcohol, and we think it is better to spray $70 \%$ alcohol multiple times instead of once. In the future, we would like to count the number of microorganisms by sterilizing by increasing the number of $70 \%$ alcohol sprays. 


\section{Conclusions}

ATP test and microbial stamp test (selective medium: general bacteria, Escherichia coli, Staphylococcus aureus, Salmonella, Vibrio parahaemolyticus) on the handle and blade of vegetable knives for the use of hygienic cooking utensils in the kitchen went. As a result, the ATP value after washing after cooking and after spraying70\% alcohol was statistically significantly lower than after washing. However, although each bacterium in the selective medium decreased, not all of them were statistically significant. In the future, after cooking, we would like to wipe off the water from the kitchen vegetable knife and then spray $70 \%$ alcohol, and then spray $70 \%$ alcohol multiple times instead of once before conducting a microbiological test.

\section{ACKNOWLEDGMENTS}

We would like to thank all the cooks who participated in this experiment. Also, we would like to thank the inspectors who also performed the ATP inspection.

\section{References Références Referencias}

1. Aycieck H, Oquz U. Karci K. Comparison of results of ATP bioluminescence and traditional ygiene swabbing methods fro the deteminaton of surface cleanliness at a hospital kitchen. (2006). Int J Hyg Environ Heatth. 209(2): 203-206.

2. Osimani A, Garofalo C, Clementi F, Tavoletti S, Aquilanti L.Bioluminescence ATP monitoring for the routine assessment fo food contact surface cleanliness in a university canteen. (2014). Int $\mathrm{J}$ Environ Res Public Health 17; 11(10): 10824-10837.

3. Lee JH (2018) An investigation of Factors that influence Hygiene Practices at a small Day Care Center. (2018). J Food Prot. 81(1): 158-164.

4. Griffith CJ, Coooper RA, Gilmore J, Davies C, Lweis $M$. An evaluation of hospital cleaning refimes and standards. (2000) J Hosp Infect. 45(1): 19-28

5. Stannard CJ, Gibbs PA. Rapid microbiology: application $s$ of bioluminescence in the food industry-a review. (1986) J Biolumin Chemilumin 1(1): 3-10.

6. Nante N, Ceriale E, Messina G, Lenzi D, Manzi P. Effectiveness of ATP bioluminescence to assess hospital cleaning: a review. (2017) J Prev. Med. Hyg. 58(2): E177-E183.

7. Amodio E, Dubi C. Use of ATP bioluminescence for assessing $\mathrm{h}$ eclealiness of hospital surfaces: a review of the published literature (1990-2012).(2014) $\mathrm{J}$ infect Public Health 7(2): 92-98

8. Stanley PE. A review of bioluminescent STP techniques in papid microbiology. (1989) J Biolumin Chemilumin 4(1): 375-380
9. Katayama N, Ito A, Hirabayashi M, Kondo S, Nakayama Y, Naka A, Sasaki N, Inuzuka M, Tamura T. Results of Hygiene Education of Kitchen Knife by using ATP Inspection - Comparison of Handle and blade. (2020) Global Journal of Medical Research. 20(5):1-6.

10. Katayama N, Hirabayashi M, Ito A, Kondo S, Nakayama Y, Naka A, Sasaki N, Inuzuka M, Tamura T. Results of Hygiene Education of Kitchen Cutting Board by using ATP Inspection - Comparison of Vegetable Cutting Board and Meat Cutting Board. (2020) Global Journal of Medical Research. 20(5):13-16.

11. Katayama N, Kondo S, Ito A, Hirabayashi $M$, Nakayama Y, Naka A, Sasaki N, Inuzuka M, Tamura T. Results of Hygiene Education of Kitchen Stove Konb and Water Faucet by using ATP Inspection. (2020) Global Journal of Medical Research. 20(5):35-38. 